\title{
Trends in the prevalence of chronic liver disease in the Korean adult population, 1998-2017
}

Seung Ha Park', Lindsay D. Plank², Ki Tae Suk³, Yong Eun Park', Jin Lee', Joon Hyuk Choi', Nae Yun Heo', Jongha Park', Tae Oh Kim', Young Soo Moon', Hyun Kuk Kim', Hang Jea Jang', Ha Young Park ${ }^{4}$, and Dong Joon Kim ${ }^{3,5}$

${ }^{1}$ Department of Internal Medicine, Inje University Haeundae Paik-Hospital, Inje University College of Medicine, Busan, Korea; ${ }^{2}$ Department of Surgery, Faculty of Medical and Health Sciences, University of Auckland, Auckland, New Zealand; ${ }^{3}$ Department of Internal Medicine, Hallym University College of Medicine, Chuncheon; ${ }^{4}$ Department of Emergency Medicine, Inje University Haeundae PaikHospital, Inje University College of Medicine, Busan; ${ }^{5}$ Institute for Liver and Digestive Diseases, Hallym University, Chuncheon, Korea

\section{Graphical Abstract}

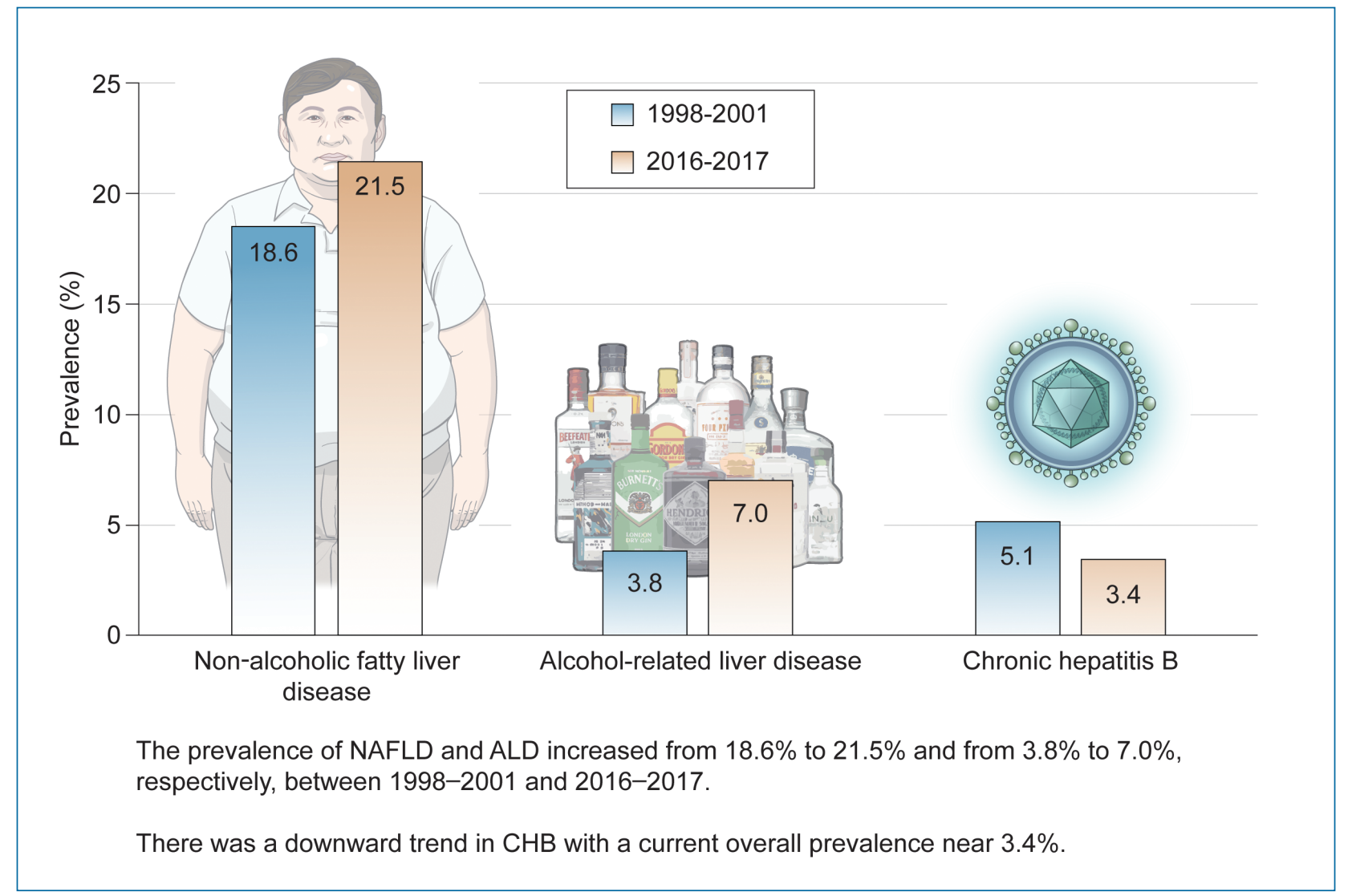

\section{Abbreviations:}

ALD, alcohol-related liver disease; ALT, alanine aminotransferase; APRI, AST-toplatelet ratio index; $\mathrm{AST}$, aspartate aminotransferase; $\mathrm{BMI}$, body mass index; $\mathrm{CHB}$ chronic hepatitis $\mathrm{B}$; $\mathrm{CHC}$, chronic hepatitis $\mathrm{C}$; Cl, confidence interval; CLD, chronic liver disease; FIB-4, fibrosis-4; HCV, hepatitis C virus; KNHANES, Korea National Health and Nutrition Examination Survey; NAFLD, non-alcoholic fatty liver disease; $\mathrm{WHO}$, world health organization

\section{Corresponding author : Dong Joon Kim}

Department of Internal Medicine, Hallym University College of Medicine, 1 Hallymdaehak-gil, Chuncheon 24252, Korea

Tel: +82-33-240-5646, Fax: +82-33-241-8064

E-mail: djkim@hallym.ac.kr

https://orcid.org/0000-0002-5792-1500 
Background/Aims: Data on the trends in the prevalence of chronic liver disease (CLD) in Korea are scarce. This study aimed to evaluate whether the CLD prevalence changed between 1998-2001 and 2016-2017.

Methods: Data were extracted from the Korea National Health and Nutrition Examination Survey (1998-2001 to 20162017; $n=25,893$ ). Non-alcoholic fatty liver disease (NAFLD) was defined as a hepatic steatosis index $>36$ in the absence of any other evidence of CLD. The definition of alcohol-related liver disease (ALD) was excessive alcohol consumption ( $\geq 210$ $\mathrm{g} /$ week for men and $\geq 140 \mathrm{~g} /$ week for women) and an ALD/NAFLD index $>0$.

Results: The prevalence of NAFLD increased from 18.6\% (95\% confidence interval [CI], 17.8-19.5\%) in 1998-2001 to $21.5 \%(95 \% \mathrm{Cl}, 20.6-22.6 \%)$ in 2016-2017. During the same time period, increases were observed in the prevalence of obesity ( 27.0 vs. $35.1 \%)$, central obesity ( 29.4 vs. $36.0 \%)$, diabetes (7.5 vs. $10.6 \%)$, and excessive drinking (7.3 vs. $10.5 \%)$. ALD prevalence also increased from $3.8 \%(95 \% \mathrm{Cl}, 3.4-4.2 \%)$ to $7.0 \%(95 \% \mathrm{Cl}, 6.4-7.6 \%)$. In contrast, chronic hepatitis B decreased from $5.1 \%(95 \% \mathrm{Cl}, 4.6-5.5 \%)$ to $3.4 \%(95 \% \mathrm{Cl}, 3.0-3.8 \%)$. The prevalence of chronic hepatitis $\mathrm{C}$ was approximately $0.3 \%$ in $2016-2017$.

Conclusions: The prevalence of NAFLD and ALD increase among Korean adults. Our results suggest potential targets for interventions to reduce the future burden of CLD. (Clin Mol Hepatol 2020;26:209-215)

Keywords: Alcoholic-related liver disease; Hepatitis B, Chronic; Hepatitis C, Chronic; Non-alcoholic fatty liver disease; Prevalence

\section{Study Highlights}

- The prevalence of NAFLD increased from 18.6\% in 1998-2001 to 21.5\% in 2016-2017.

- The prevalence of ALD increased from 3.8\% to $7.0 \%$.

- The prevalence of chronic hepatitis B decreased from $5.1 \%$ to $3.4 \%$

- The prevalence of chronic hepatitis C was approximately 0.3\% in 2016-2017.

- The prevalence of NAFLD and ALD has increased among Korean adults.

\section{INTRODUCTION}

According to global health estimates 2015 of the World Health Organization (WHO), approximately 1.2 million people worldwide die each year from cirrhosis and subsequent complications and 800,000 die of hepatocellular carcinoma, comprising $3.5 \%$ of all deaths worldwide. ${ }^{1,2}$ Especially, the Asia-Pacific region accounts for $45 \%$ of the world's deaths due to liver disease. As chronic liver disease (CLD) may develop due to a variety of etiologies including chronic viral infection, alcohol, and metabolic causes, the implement of population-based interventions that screen for the underlying cause and the use of lifestyle and pharmacological interventions to prevent or delay the progression to life-threatening cirrhosis complications and liver cancer is imperative.

The WHO recently proposed a strategic plan consisting of global efforts to eradicate hepatitis virus, the main cause of liver disease. $^{3}$ Despite ongoing efforts, the global burden of CLD is anticipated to increase in a generation. ${ }^{2}$ More than $5 \%$ of all deaths worldwide are attributed to the harmful use of alcohol, and alcohol-related liver disease (ALD) accounts for one quarter of all al- cohol-related deaths. ${ }^{4}$ Considering the increase in per capita alcohol consumption in the Asia-Pacific region, ${ }^{5}$ the prevalence of ALD and its complications is expected to increase further in the near future. Non-alcoholic fatty liver disease (NAFLD), the most notable liver disease, has emerged as a new management subject as viral liver disease enters a genuine phase. ${ }^{6}$ NAFLD is also expected to increase as the incidence of obesity increase due to westernized eating habits, a lack of exercise, and lifestyle changes. ${ }^{7}$

Over the past three decades, there have been great successes in CLD management, particularly in viral hepatitis, as well as epidemiological changes. However, data on trends in the prevalence of CLD in Korea are sparse. Recently, NAFLD modeling is being actively attempted to forecast the disease burden using currently available data. ${ }^{7}$ A nationwide prevalence investigation would provide more accurate rates in entire population and specific subpopulations $^{8}$ for use as basic data for CLD modeling.

Accordingly, we sought to determine the current prevalence of CLD in Korean adults aged 19 and older and whether the prevalence changed between 1998-2001 and 2016-2017 in a representative Korean adult population data from the Korea National 
Health and Nutrition Examination Survey (KNHANES).

\section{MATERIALS AND METHODS}

\section{Sample population}

The KNHANES is a series of cross-sectional national health and nutrition surveys designed to provide representative prevalence estimates for a variety of health measures and conditions. The KNHANES is conducted by the Korean Centers for Disease Control. The survey design is a complex, stratified, multi-stage probability sampling of the civilian, non-institutionalized Korean population. The procedures used to select the sample as well as to conduct the interviews and examinations have been specified elsewhere. ${ }^{9}$ This survey included an interview to obtain information concerning an individual's health history, health behaviors, and risk factors. A subsequent health examination was performed at a mobile examination center.

Our analyses included data from 1998-2001 (KNHANES I and II) and 2016-2017 (KNHANES VII-1 and VII-2). We included a total of 14,801 participants aged 19 years and older from KNHANES 1998-2001 and 12,900 participants from KNHANES 2016-2017, respectively in this study. Of that number, 1,808 subjects who had incomplete data on NAFLD prediction model or biochemical/clinical parameters were excluded. Finally, 25,893 (14,438 in KNHANES 1998-2001 and 11,455 in KNHANES 2016-2017) participants were included in the analyses. Informed consent was obtained from all participants, and the protocol was approved by the Institutional Review Board of the Korean Centers for Disease Control.

\section{Definition of CLD etiology}

NAFLD was defined using the validated hepatic steatosis index which was calculate as $8 \times$ alanine aminotransferase / aspartate aminotransferase (ALT / AST) ratio + body mass index (BMI) $(+2$, if diabetes; +2 , if female $)^{10}$ in the absence of any other evidence of CLD, such as excessive alcohol intake or a positive hepatitis $B$ or hepatitis C test. From 1998-2001, since anti-hepatitis C virus (anti-HCV) tests were not performed in KNHANES, chronic hepatitis $\mathrm{C}(\mathrm{CHC})$ cannot be ruled out in defining NAFLD during that period. The optimal cut-off value for NAFLD was set at $>36$. In the subpopulation with NAFLD, the AST-to-platelet ratio index $(A P R I)^{11}$ and fibrosis-4 (FIB-4) $)^{12}$ were used to evaluate liver fibrosis. Their formula were as follows: APRI $=[($ AST / upper limit of normal) / platelet count $\left.\left(10^{9} / \mathrm{L}\right)\right] \times 100$, where the upper limits of normal AST levels were set at $37 \mathrm{IU} / \mathrm{L}$ for men and $29 \mathrm{IU} / \mathrm{L}$ for women, ${ }^{13} \mathrm{FIB}-4=$ age $\times$ AST $/$ [platelets in $10^{9} / \mathrm{L} \times(\mathrm{ALT})^{1 / 2}$ ]. Cutoff values for advanced fibrosis $(\geq F 3)$ were set at 1 of $A P R I$ and 2.67 of FIB-4. APRI and FIB-4 were calculated only in KNHANES 2016-2017 due to a lack of data in the KNHANES 1998-2001.

Chronic hepatitis B (CHB) was defined as HBsAg seropositivity. An anti-HCV test was done only in the KNHANES 2016-2017, but an HCV RNA polymerase chain reaction (PCR) test was not performed. We assumed that CHC affected approximately $30 \%$ of those with anti-HCV positivity based on an observation in KNHANES 2013-2015, in which an HCV RNA PCR test showed that 37 individuals were HCV RNA positive among 119 subjects with anti-HCV positivity. The study definition of ALD was a com-

Table 1. Demographic and clinical characteristics of the study sample

\begin{tabular}{|c|c|c|c|}
\hline Characteristic & 1998-2001 & 2016-2017 & $P$-value \\
\hline Total & 14,438 & 11,455 & \\
\hline \multicolumn{4}{|l|}{ Gender (\%) } \\
\hline Male & 49.0 & 50.2 & 0.046 \\
\hline Female & 51.0 & 49.8 & \\
\hline Age (years) & 42.2 & 46.8 & $<0.001$ \\
\hline \multicolumn{4}{|l|}{ Smoking (\%) } \\
\hline Never & 58.2 & 56.6 & $<0.001$ \\
\hline Former & 8.8 & 21.5 & \\
\hline Current & 33.0 & 21.9 & \\
\hline Excessive drinking (\%) & 7.3 & 10.5 & $<0.001$ \\
\hline Obesity (\%) & 27.0 & 35.1 & $<0.001$ \\
\hline Central obesity (\%) & 29.4 & 36.0 & $<0.001$ \\
\hline Diabetes (\%) & 7.5 & 10.6 & $<0.001$ \\
\hline Hypertension (\%) & 22.6 & 27.1 & $<0.001$ \\
\hline Hypercholesterolemia (\%) & 7.7 & 19.8 & $<0.001$ \\
\hline TyG index & 4.64 & 4.65 & 0.005 \\
\hline Hepatic steatosis index & 32.0 & 32.8 & $<0.001$ \\
\hline NAFLD (\%) & 18.6 & 21.5 & $<0.001$ \\
\hline $\begin{array}{l}\text { Alcohol-related liver } \\
\text { disease (\%) }\end{array}$ & 3.8 & 7.0 & $<0.001$ \\
\hline Chronic hepatitis B (\%) & 5.1 & 3.4 & $<0.001$ \\
\hline Chronic hepatitis C (\%) & NA & 0.3 & \\
\hline
\end{tabular}

Values are presented as weighted percentages or means. Excessive drinking: $\geq 210 \mathrm{~g} /$ week for men and $\geq 140 \mathrm{~g} /$ week for women; obesity: body mass index $\geq 25$; central obesity: waist circumference $\geq 90 \mathrm{~cm}$ for men and $\geq 80$ $\mathrm{cm}$ for women.

TyG index, triglycerides and glucose index; NAFLD, non-alcoholic fatty liver disease. 
bination of excessive alcohol consumption ( $\geq 210 \mathrm{~g} /$ week for men and $\geq 140 \mathrm{~g} /$ week for women) and an ALD/NAFLD index $>0$, which was calculated as $-58.5+0.637$ (mean corpuscular volume) + 3.91 (AST/ALT) -0.406 (BMI) + 6.35 for male gender. ${ }^{14}$

Table 2. Gender-specific prevalence of chronic liver disease

\begin{tabular}{lcc}
\hline Type of disease & 1998-2001 & 2016-2017 \\
\hline NAFLD & 18.7 & 23.7 \\
Male & 18.6 & 19.3 \\
$\quad$ Female & & \\
Alcohol-related liver disease & 7.5 & 12.3 \\
Male & 0.2 & 1.7 \\
Female & & \\
Chronic hepatitis B & 5.7 & 3.5 \\
Male & 4.4 & 3.2 \\
Female & & \\
Chronic hepatitis C & NA & 0.4 \\
Male & NA & 0.2 \\
Female &
\end{tabular}

Values are presented as weighted percentages.

NAFLD, non-alcoholic fatty liver disease.
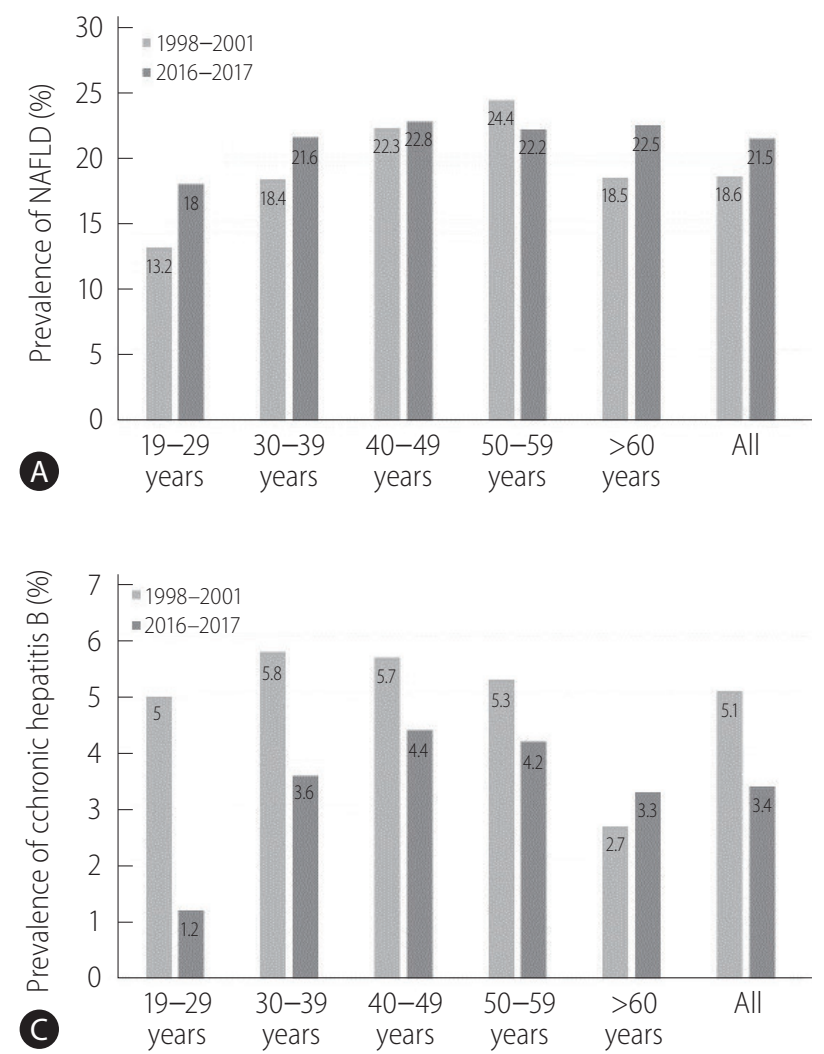

\section{Definition of clinical parameters}

Diabetes mellitus was defined based on use insulin or oral hypoglycemic agents or fasting plasma glucose $\geq 126 \mathrm{mg} / \mathrm{dL}$. Participants were diagnosed as hypertensive if the systolic pressure was $\geq 140 \mathrm{mmHg}$, diastolic pressure was $\geq 90 \mathrm{mmHg}$, or antihypertensive medication was used. Hypercholesterolemia was defined as a cholesterol level $\geq 240 \mathrm{mg} / \mathrm{dL}$. Obesity was defined as a BMI of $\geq 25{ }^{15}$ while central obesity was defined using the thresholds for waist circumference ( $\geq 90 \mathrm{~cm}$ for men and $\geq 80 \mathrm{~cm}$ for women). ${ }^{16}$ Insulin resistance was calculated using the triglycerides and glucose index as follows: $\mathrm{Ln}$ [fasting glucose $(\mathrm{mg} / \mathrm{dL}) \times$ triglycerides $(\mathrm{mg} / \mathrm{dL}) / 2)^{17}$

\section{Data analyses}

Sample weights were included in the estimation process for all of the analyses to reflect the differential probabilities for selection, non-response, and non-coverage. ${ }^{9}$ All analyses were performed using Complex Samples in SPSS statistics (version 25.0; IBM Corp., Armonk, NY, USA), which provides the specialized statistics for complex sample designs, such as stratified, clustered or

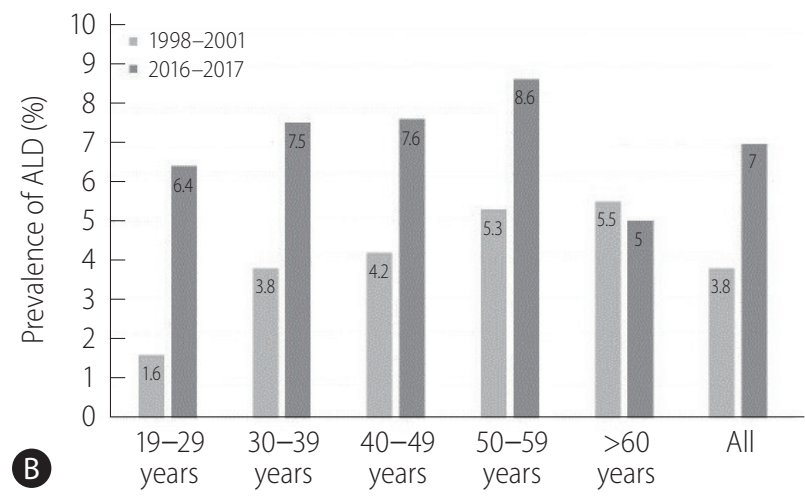

Figure 1. Prevalence of CLD by age groups on KNHANES in 1998-2001 and 2016-2017. Data are reported as weighted percentages. (A) NAFLD prevalence, (B) ALD prevalence, (C) CHB prevalence. CLD, chronic liver disease; NAFLD, non-alcoholic fatty liver disease; ALD, alcohol-related liver disease; $\mathrm{CHB}$, chronic hepatitis B. 
multistage sampling. When merging KNHANES survey cycles, appropriate selection of sampling weights and adjustment coefficients were applied according to the KNHANES guidelines. ${ }^{18}$ The data were presented as weighted means or weighted proportions with standard errors for continuous or categorical variables, respectively. Differences between the two study cycles were examined with Wald $\mathrm{F}$ test statistics in a general linear model for continuous variables and the Rao-Scott adjusted chi-square test for categorical variables.

\section{RESULTS}

Table 1 shows the weighted distribution of the KNHANES 1998-2001 and 2016-2017 samples. With the exception of smoking status and CHB, all clinical parameters disadvantageously changed during two decades. Compared with 1998-2001, the prevalence in 2016-2017 increased for obesity, diabetes, and hypertension. These unfavorable changes represented with an increased NAFLD prevalence in 2016-2017 from 18.6\% (95\% confidence interval $[\mathrm{Cl}], 17.8-19.5 \%)$ in 1998-2001 to $21.5 \%$ (95\% $\mathrm{Cl}, 20.6-22.6 \%)$. Along with increase in excessive drinking, the prevalence of ALD also increased from 3.8\% (95\% Cl, 3.4-4.2\%) in $1998-2001$ to $7.0 \%(95 \% \mathrm{Cl}, 6.4-7.6 \%)$ in $2016-2017$. In con-

Table 3. Prevalence of NAFLD by selected characteristics

\begin{tabular}{lcc}
\hline Characteristic & 1998-2001 & 2016-2017 \\
\hline Obesity & 5.3 & 4.1 \\
No & 54.2 & 53.7 \\
Yes & & \\
Central obesity & 7.7 & 6.4 \\
No & 44.7 & 48.4 \\
Yes & & \\
Diabetes & 16.4 & 18.3 \\
No & 46.0 & 49.4 \\
Yes & & \\
Hypertension & 15.8 & 17.9 \\
No & 28.3 & 31.5 \\
Yes & & \\
Hypercholesterolemia & 17.0 & 18.7 \\
No & 36.8 & 32.8 \\
Yes & & \\
\hline
\end{tabular}

Values are presented as weighted percentages.

NAFLD, non-alcoholic fatty liver disease. trast, the prevalence of CHB in 2016-2017 decreased from 5.1\% (95\% Cl, 4.6-5.5\%) in 1998-2001 to 3.4\% (95\% Cl, 3.0-3.8\%) in 2016-2017. The prevalence of CHC was approximately $0.3 \%$ in 2016-2017. Gender-specific trends in the prevalence of CLD are presented in Table 2. Changes in the prevalence of NAFLD, ALD, and $\mathrm{CHB}$ during the study period were evident in both genders.

Figure 1 shows the changes in the prevalence of NAFLD, ALD, and CHB. The increase in the prevalence of NAFLD was led by the 20s, 30s, and 60s age groups. With the exception of the 60s group, increases in ALD prevalence were seen in all age strata. There was a sharp increase in ALD prevalence in 20s from 1.6\% (95\% Cl, 1.1-2.3\%) in 1998-2001 to 6.4\% (95\% Cl, 4.9-8.3\%) in 2016-2017. The prevalence of CHB was decreased in all age groups except in the 60s. In particular, the prevalence decreased dramatically in the 20 s from $5.0 \%(95 \% \mathrm{Cl}, 4.1-6.2 \%)$ in $1998-$ 2001 to $1.2 \%(95 \% \mathrm{Cl}, 0.7-2.0 \%)$ in 2016-2017. A similar trend was observed in the 30 s from $5.8 \%(95 \% \mathrm{Cl}, 5.1-6.7 \%)$ in 1998-2001 to 3.6\% (95\% Cl, 2.7-4.8\%) in 2016-2017.

When the study sample was stratified by selected characteristics, the prevalence of NAFLD was approximately 50\% among participants with obesity and diabetes and 30\% among those with hypertension and hypercholesterolemia in both survey cycles (Table 3). Among the 2,440 participants with NAFLD in 20162017, the prevalence of advanced fibrosis, defined by an APRI $>1$ and an FIB-4 $>2.67$, were $1.4 \%(95 \% \mathrm{Cl}, 0.9-2.1 \%)$ and $1.2 \%$ $(95 \% \mathrm{Cl}, 0.8-1.8 \%)$, respectively.

\section{DISCUSSION}

The current study provides an estimate of the current and past prevalence of CLD in Korean adult population and demonstrates how the prevalence has changed over two decades using KNHANES surveys. The KNHANES is the only population-based survey that provides nationally representative estimates of the prevalence -and, therefore, the lifetime risk- of CLD. Consequently, relatively accurate, clinically relevant population estimates can be generated, and time trends can be identified. The two surveys described here indicated that the prevalence of NAFLD and ALD increased by $16 \%$ and $84 \%$, respectively, in persons aged 19 years or older over the 19-year interval. In contrary, we found encouraging results with regard to the prevalence of $\mathrm{CHB}$ which decreased by $33 \%$.

From our analyses, we estimate that the prevalence of NAFLD increased from $18.6 \%$ in $1998-2001$ to $21.5 \%$ in $2016-2017$. 
This increase was evident in age groups of 20s, 30s, and 60s as well as in both genders. This increase may be large due to the increasing population of obesity and diabetes in which the prevalence of NAFLD is approximately $50 \%$. It also could be attributable to the aging Korean population (42 years in 1998-2001 vs. 47 years in 2016-2017). This increasing trend of NAFLD prevalence reported here is similar to that of a recent systematic review of an Asian population. ${ }^{19}$ Another study predicted that NAFLD prevalence in Asia will increase from 18\% in 2016 to $22 \%$ by 2030 and that obesity will increase from 27\% in 2016 to $28.5 \%$ by $2030 .{ }^{8}$ Taken together, our results demonstrate that unless there is apparent attenuation in the trend of well-established risk factors, the prevalence of NAFLD is expected to continually increase. We also found that $1.2-1.5 \%$ of the participants with NAFLD had advanced fibrosis $(\geq F 3)$. Its prevalence is similar to previously reported estimates in US population, ${ }^{20}$ representing a major disease burden in now and near future.

As expected by the observed increase in excessive alcohol consumption, the estimated prevalence of ALD increased approximately $85 \%$ over this same period. This sharp increasing trend in ALD was primarily driven by increasing ALD levels in young adults. Our analysis identified a strong and upward trend in ALD prevalence among person younger than 40 years. Considering that the early initiation of alcohol abuse in early adulthood is associated with unhealthy drinking patterns and adverse psychosocial, behavioral, and other long-term health outcomes, ${ }^{21}$ this unfavorable trend of excessive consumption corresponding with ALD in young adults is a concern from a public health perspective.

Our results shed some light on encouraging trends in CHB. We observed a downward trend in CHB across all age groups, with the exception of the $60 \mathrm{~s}$. In the 20s age group, we revealed that the prevalence of CHB fell from $5 \%$ in $1998-201$ to $1.2 \%$ in 2016-2017. Because CHB is a lifetime infectious disease, any increases or decreases in CHB are expected to be first evident in younger persons. This finding is in agreement with a previous study, which reported that the incidence and prevalence of CHB decreased over the last two decades due to the successful implementation of a vaccination program. ${ }^{22,23}$ Despite the substantial progress in preventing CHB in young adults, this study confirms the ongoing high prevalence of $\mathrm{CHB}$ among Korean adults. The consequent burden of $\mathrm{CHB}$ remains high and is consistent with findings from a recent modelling study. ${ }^{24}$

Our findings of the prevalence of anti-HCV positivity $(0.8 \%$; $95 \% \mathrm{Cl}, 0.6-1.0 \%)$ are consistent with a prior analysis of Korean data in 2009 that reported $0.8 \%$ of about 300,000 health-check examinees. ${ }^{25}$ Our study extended the temporal trend analysis through 2017 to demonstrate continued stability of anti-HCV positivity. Based on a finding that one third resulted in HCV RNA positivity among those with anti-HCV positivity, the extrapolation of KNHANES data suggests a $0.3 \%$ CHC prevalence in 2016 2017. The estimated prevalence of $\mathrm{CHC}$ reported here is much lower than previous estimates (approximately 1\%) in Korea or a recent global estimate, ${ }^{26,27}$ which was based on anti-HCV positivity.

The main strength of the current study is that the data were representative of the Korean population and collected using standardized measurements. Although this study makes important contributions to the literature, it also has a number of limitations. First, because of the absence of imaging or histological diagnosis of NAFLD and ALD, we adopted operational criteria for defining NAFLD and ALD based on a predictive model that has been validated. Therefore, interpretation of our results should be made with caution. Second, the data on alcohol measurement were based on self-reports; thus, they may be influenced by recall and social desirability bias. Third, rather than tracing the changes that occurred over decades, our study simply compared the prevalence at two time points. Although we can confirm an increase in NAFLD and ALD during the study period, it is unclear whether the prevalence of the two diseases was continuously increasing. Finally, the survey participants were sampled only from the non-institutionalized Korean population. The following persons were excluded from the analysis: incarcerated persons, homeless persons, and person who were physically unable to undergo the survey. Therefore, the overall CLD prevalence might be underestimated.

In conclusion, our study found that the prevalence of NAFLD and ALD, particularly in young adults, increased in the Korean adult population from $18.6 \%$ to $21.5 \%$ and from $3.8 \%$ to $7.0 \%$, respectively, between 1998-2001 and 2016-2017. However, there is a downward trend in CHB with a current overall prevalence near $3.4 \%$. The $\mathrm{CHC}$ prevalence appears to have plateaued $0.3 \%$. Our results demonstrate potential targets for interventions to reduce the future burden of CLD. Public health efforts are required to prevent and improve modifiable risk factors of CLD.

\section{Author's contribution}

Study concept and design: S.H.P., L.D.P., K.T.S., Y.S.M, and D.J.K.; Data collection, statistical analysis, and interpretation of results: S.H.P., Y.E.P., J.L., J.H.C., N.Y.H., J.P., T.O.K., H.K.K., H.J.J.; Writing of article: S.H.P., H.Y.P., D.J.K. 


\section{Conflicts of Interest}

The authors have no conflicts to disclose.

\section{REFERENCES}

1. World Health Organization (WHO). Global health estimates 2015: deaths by cause, age, sex, by country and by region, 2000-2015. Geneva: WHO, 2016.

2. Asrani SK, Devarbhavi H, Eaton J, Kamath PS. Burden of liver diseases in the world. J Hepatol 2019;70:151-171.

3. World Health Organization (WHO). Global status report on alcohol and health 2018. Geneva: WHO, 2018.

4. Fan JG, Kim SU, Wong VW. New trends on obesity and NAFLD in Asia. J Hepatol 2017;67:862-873.

5. Jang JY, Kim DJ. Epidemiology of alcoholic liver disease in Korea. Clin Mol Hepatol 2018;24:93-99.

6. Yoo JJ, Kim W, Kim MY, Jun DW, Kim SG, Yeon JE, et al. Recent research trends and updates on nonalcoholic fatty liver disease. Clin Mol Hepatol 2019;25:1-11.

7. Estes C, Anstee QM, Arias-Loste MT, Bantel H, Bellentani S, Caballeria J, et al. Modeling NAFLD disease burden in China, France, Germany, Italy, Japan, Spain, United Kingdom, and United States for the period 2016-2030. J Hepatol 2018;69:896-904.

8. Li Z, Xue J, Chen P, Chen L, Yan S, Liu L. Prevalence of nonalcoholic fatty liver disease in mainland of China: a meta-analysis of published studies. J Gastroenterol Hepatol 2014;29:42-51.

9. Kweon S, Kim Y, Jang MJ, Kim Y, Kim K, Choi S, et al. Data resource profile: the Korea National Health and Nutrition Examination Survey (KNHANES). Int J Epidemiol 2014;43:69-77.

10. Lee JH, Kim D, Kim HJ, Lee CH, Yang JI, Kim W, et al. Hepatic steatosis index: a simple screening tool reflecting nonalcoholic fatty liver disease. Dig Liver Dis 2010;42:503-508.

11. Wai CT, Greenson JK, Fontana RJ, Kalbfleisch JD, Marrero JA, Conjeevaram HS, et al. A simple noninvasive index can predict both significant fibrosis and cirrhosis in patients with chronic hepatitis $C$. Hepatology 2003;38:518-526.

12. Vallet-Pichard A, Mallet $V$, Nalpas B, Verkarre V, Nalpas A, DhalluinVenier $V$, et al. FIB-4: an inexpensive and accurate marker of fibrosis in HCV infection. comparison with liver biopsy and fibrotest. Hepatology 2007;46:32-36.

13. Park SH, Heo NY, Kim CH, Suk KT, Kim DJ, Lee HY. Upper reference limits for aminotransferase activities and the prevalence of elevated aminotransferase activities in a Korean population. J Clin Gastroenterol 2013;47:76-82.

14. Dunn W, Angulo P, Sanderson S, Jamil LH, Stadheim L, Rosen C, et al. Utility of a new model to diagnose an alcohol basis for steato- hepatitis. Gastroenterology 2006;131:1057-1063.

15. WHO Expert Consultation. Appropriate body-mass index for Asian populations and its implications for policy and intervention strategies. Lancet 2004;363:157-163.

16. Alberti KG, Eckel RH, Grundy SM, Zimmet PZ, Cleeman JI, Donato $K A$, et al. Harmonizing the metabolic syndrome: a joint interim statement of the International Diabetes Federation Task Force on Epidemiology and Prevention; National Heart, Lung, and Blood Institute; American Heart Association; World Heart Federation; International Atherosclerosis Society; and International Association for the Study of Obesity. Circulation 2009;120:1640-1645.

17. Guerrero-Romero F, Simental-Mendía LE, González-Ortiz M, Martínez-Abundis E, Ramos-Zavala MG, Hernández-González SO, et al. The product of triglycerides and glucose, a simple measure of insulin sensitivity. Comparison with the euglycemic-hyperinsulinemic clamp. J Clin Endocrinol Metab 2010;95:3347-3351.

18. Korea Centers fo Disease Control \& Prevention (KCDC). Korea National health and nutrition examination survey: analytic guidelines. KCDC web site, <https://knhanes.cdc.go.kr/knhanes/sub03/ sub03_06_02.do>. Accessed 7 May 2019.

19. Li J, Zou B, Yeo YH, Feng Y, Xie X, Lee DH, et al. Prevalence, incidence, and outcome of non-alcoholic fatty liver disease in Asia, 1999-2019: a systematic review and meta-analysis. Lancet Gastroenterol Hepatol 2019;4:389-398.

20. Kabbany MN, Conjeevaram Selvakumar PK, Watt K, Lopez R, Akras Z, Zein N, et al. Prevalence of nonalcoholic steatohepatitisassociated cirrhosis in the United States: an analysis of national health and nutrition examination survey data. Am J Gastroenterol 2017;112:581-587.

21. Fan AZ, Russell M, Stranges S, Dorn J, Trevisan M. Association of lifetime alcohol drinking trajectories with cardiometabolic risk. J Clin Endocrinol Metab 2008;93:154-161.

22. Hyun Kim B, Ray Kim W. Epidemiology of hepatitis B virus infection in the United States. Clin Liver Dis (Hoboken) 2018;12:1-4.

23. Park SH. Trends in the seroprevalence of hepatitis B surface antigen in the South Korean population. Int J Infect Dis 2012;16:e669-e672.

24. Polaris Observatory Collaborators. Global prevalence, treatment, and prevention of hepatitis B virus infection in 2016: a modelling study. Lancet Gastroenterol Hepatol 2018;3:383-403.

25. Kim DY, Kim IH, Jeong SH, Cho YK, Lee JH, Jin YJ, et al. A nationwide seroepidemiology of hepatitis $\mathrm{C}$ virus infection in South Korea. Liver Int 2013;33:586-594.

26. Suh DJ, Jeong SH. Current status of hepatitis C virus infection in Korea. Intervirology 2006:49:70-75.

27. Polaris Observatory HCV Collaborators. Global prevalence and genotype distribution of hepatitis C virus infection in 2015: a modelling study. Lancet Gastroenterol Hepatol 2017;2:161-176. 